\title{
Recent VLBI Results on SN 1986J and the Possibility of FRBs Originating from Inside the Expanding Ejecta of Supernovae
}

\author{
Michael Bietenholz ${ }^{* \dagger}$ \\ SARAO, South Africa \\ E-mail: michael@hartrao.ac.za
}

\section{Norbert Bartel}

York University Toronto, Canada

\begin{abstract}
We discuss our VLA and VLBI observations of supernova 1986J, which is characterized by a compact radio-bright component within the expanding shell of ejecta. No other supernova (SN) has such a central component at $\mathrm{cm}$ wavelengths. The central component therefore provides a unique probe of the propagation of radio signals at $\mathrm{cm}$ wavelengths through the ejecta of a young SN. Such a probe is important in the context of Fast Radio Bursts (FRB), which, in many models, are thought to be produced by young magnetars or neutron stars. The FRB signals would therefore have to propagate through the expanding SN ejecta. Our observations of the Type II SN 1986J show that its ejecta will remain opaque to $\mathrm{cm}$-wave emission like FRBs for at least several decades after the explosion, and by the time the ejecta have become transparent, the contribution of the ejecta to the dispersion measure is likely small.
\end{abstract}

14th European VLBI Network Symposium \& Users Meeting (EVN 2018)

8-11 October 2018

Granada, Spain

\footnotetext{
*Speaker.

${ }^{\dagger}$ Also at York University, Toronto, Canada.
} 


\section{Introduction}

Fast Radio Bursts (FRBs) are bursts of radio emission, 0.1 to $10 \mathrm{Jy}$ at $\sim 1 \mathrm{GHz}$, which occur on timescales of milliseconds or less. They are characterized by high dispersion measures, $\mathrm{DM}=$ $\int N_{e} \cdot d l$, in the range of 200 to $2000 \mathrm{~cm}^{-3} \mathrm{pc}$. Their origin is still mysterious (for recent reviews, see e.g. [1,2], and also Marcote this issue). These DMs are far higher than expected from our own Galaxy's interstellar medium. If the DM is interpreted as being due to the intergalactic medium, cosmological distances are implied, with redshifts, $z$ in the range of 0.2 to 1.6. Indeed the single FRB which has so far been accurately localized, FRB 121102, was found to be at $z=0.129$ [3].

Many of the models of FRBs have them originate in young pulsars or magnetars (see, e.g. [1]). A young pulsar or magnetar would have been born in a supernova ( $\mathrm{SN}$ ) explosion, and therefore would still be embedded in an expanding cloud of SN ejecta. The FRB signals would then have to propagate through the SN ejecta to reach us. These ejecta, expected to be partly ionized, are possibly the source of the large DMs.

It is difficult, however, to obtain any direct observational constraints on the propagation of radio signals through the ejecta of a young SN. SN 1986J is a unique case where we can obtain such observational constraints. Its age, at present, is $t \sim 35 \mathrm{yr}^{1}$. It is relatively nearby, in the galaxy NGC 891, at a distance of 10 Mpc (NASA/IPAC Extragalactic Database, NED). SN 1986J is bright in the radio, and we have been observing it with very long baseline interferometry (VLBI) since 1987 and can resolve the expanding ejecta [4, 5, 6, 7, 8]. We show a dual-frequency VLBI image of SN 1986J in Figure 1. A singular feature of SN 1986J, not seen in any other SN at cm wavelengths, is a compact radio-emitting component which appears to be inside the expanding shell of ejecta. This component's central location, its longevity and its high brightness argue strongly that it is in fact near the physical centre of the expanding ejecta and not just central in projection [7].

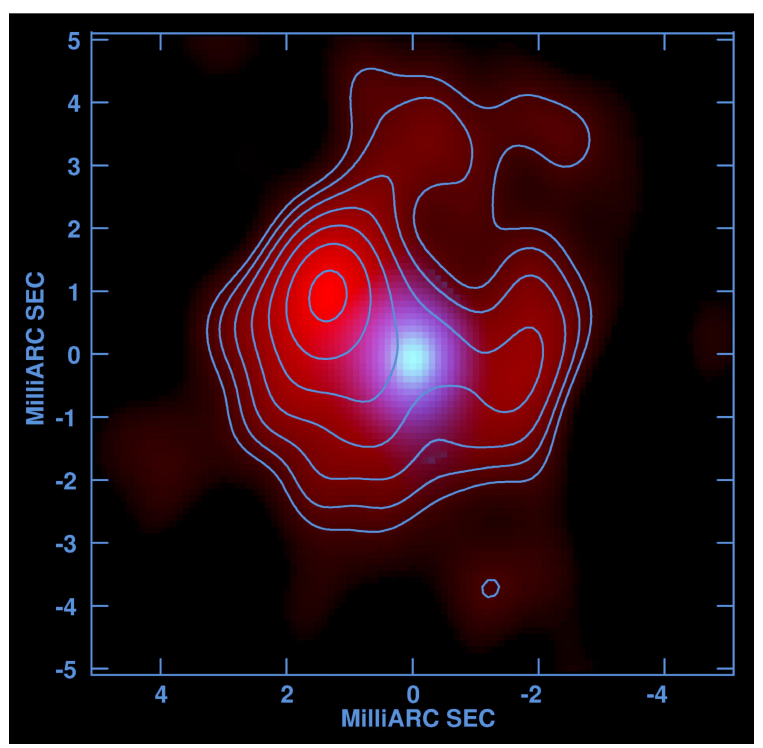

Figure 1: A phase-referenced dual-frequency VLBI image of SN 1986J from data taken 2002 to 2003, showing the compact, inverted-spectrum component located almost precisely in the projected centre of the expanding shell. The red colour and the contours represent the $5 \mathrm{GHz}$ radio brightness, showing the shell emission. The contours are drawn at $11.3,16,22.6, \ldots, 90.5 \%$ of the peak $5 \mathrm{GHz}$ brightness of $0.55 \mathrm{mJy}$ beam $^{-1}$. The blue through white colours show the $15 \mathrm{GHz}$ radio brightness distribution, which is dominated by the compact, central component which appeared around 1999. North is up and east to the left. For details see [9].

The nature of this central component is not yet clear. SN 1986J's progenitor was sufficiently massive [10] that either a neutron-star or black-hole compact remnant may have formed. The cen-

\footnotetext{
${ }^{1}$ Although SN 1986J was first detected in 1986, the explosion was a few years earlier, in 1983 [4].
} 
tral component may therefore represent radio emission either from a nascent pulsar-wind nebula or from accretion onto the newly-formed black hole. It may, however, also represent shock interaction with a very aspherical circumstellar medium distribution left over from a binary progenitor which had undergone a period of common-envelope evolution. We discuss the various hypotheses in [7]. However, for the present purpose, the physical nature of the central component is not important, the important thing is only that it resides within the expanding cloud of SN ejecta.

No FRB has been observed from SN 1986J, although we note that if one had occurred, the odds that it would have been detected are quite low. However, the central component produces radio emission at $\sim 1 \mathrm{GHz}$, which would be affected by the intervening ejecta in much the same way as that from an FRB would be. What then can we say about the propagation of FRB-like radio emission through the ejecta of a young SN on the basis of our observations of SN 1986J?

\section{Observations}

We describe our observations briefly here. More detail can be found in our series of papers $[4,5,6,7]$. We have observed SN 1986J with the Jansky Very Large Array (VLA), to measure the evolving total flux density at a wide range of frequencies. These observations allow us to determine the evolution of the radio spectral energy distribution (SED) with time. Other VLA observations were also obtained earlier by [11]. We further obtained VLBI observations at a number of epochs between 1987 and 2014, mostly using a global array consisting of antennas from the European VLBI Network (EVN) and the National Radio Astronomy Observatory (NRAO; USA). The most recent VLBI observations were obtained in 2014 Oct., at $5.0 \mathrm{GHz}$ using a global array of EVN and NRAO antennas (see [6]).

\section{Evolution of the Spectral Energy Distribution}

We show the evolution of the SED in Figure 2. At low frequencies, the SED has the form of the simple powerlaw typically seen for SNe, with spectral index, $\alpha \simeq-0.5$ (where $S_{v} \propto v^{\alpha}$, where $v$ is the frequency). This is the radio emission due to the expanding shell, which is optically thin. At high frequencies, the radio emission is dominated by that of the central component, and is still partly absorbed. An inversion appears in the SED at the frequency where the central component is becoming prominent. At yet higher frequency, the central component also becomes optically thin, and the spectrum again turns over. The two inflection points (inversion point and high-frequency turnover) both move downward in frequency as a function of time, as can be seen in Fig. 2 .

In the latest (bottom) SED curve in Fig. 2, at $t=30 \mathrm{yr}$, the emission from the central component is absorbed below a turnover frequency of $v_{\text {peak }} \simeq 15 \mathrm{GHz}$, with the absorption being due to the free-free mechanism [4]. At $t=30 \mathrm{yr}$, therefore, any signal at $1 \mathrm{GHz}$, such as an FRB, would still be strongly absorbed by the SN ejecta. If FRBs originate in young SNe similar to SN 1986J, we would expect not to see them for at least several decades after the explosion.

We can estimate the time until transparency at $1 \mathrm{GHz}$ for SN 1986J as follows. The optical depth to free-free emission is given by:

$$
\tau=3.28 \times 10^{-7}\left(\frac{v}{\mathrm{GHz}}\right)^{-2.1}\left(\frac{T_{e}}{10^{4} \mathrm{~K}}\right)^{-1.35}\left(\frac{\mathrm{EM}}{\mathrm{cm}^{-6} \mathrm{pc}}\right),
$$


where $T_{e}$ is the electron temperature, which we take to the $10^{4} \mathrm{~K}$, and $\mathrm{EM}=\int N_{e}^{2} d l$, is the emission measure, where $N_{e}$ is the number density of free electrons, and $l$ is the path-length along the line of sight from the centre of the $\mathrm{SN}$ to the observer.

The turnover frequency is approximately that where $\tau_{v}=1$. For SN $1986 \mathrm{~J}$ at $t=30 \mathrm{yr}, v_{\text {peak }}=$ $15 \mathrm{GHz}$. We can then calculate that $\mathrm{EM} \simeq 9 \times 10^{8} \mathrm{~cm}^{-6} \mathrm{pc}$. The SED however is not static, but rather is evolving in time. In particular $v_{\text {peak }}$ is decreasing with time as can be seen in Fig. 2. To characterize the evolving SED of SN 1986J, we fitted a model with 8 free parameters to the flux density measurements (such as those in Fig. 2) between $t=14$ and $30 \mathrm{yr}$ using Bayesian statistics.

Our model has two parts: $(a)$ the shell, which is optically thin and whose flux density is a power-law function of time, and $(b)$, the central component, whose intrinsic flux density is also a power-law function of time, but which suffers time-dependent absorption by material whose emission measure, EM, is a power-law function of time. Both parts are assumed to have an intrinsic spectrum (before any absorption) of power-law form. The eight free parameters of the model are: the two intrinsic flux densities and the EM at $t=20 \mathrm{yr}$, the two optically-thin spectral indices, and the powerlaw index of the time dependencies of the two flux densities and of the EM with time.

For details of the fitted values, and other results, please see [7, 12]. Of interest in our context of FRB signals propagating through the ejecta is the solution for EM:

$$
\mathrm{EM}=(1.64 \pm 0.21) \times 10^{9}(t / 20 \mathrm{yr})^{-2.72 \pm 0.26} \mathrm{~cm}^{-6} \mathrm{pc} .
$$

The EM, and thus the absorption, are decreasing with time. This is expected as the SN is expanding. In a homologously expanding system with a constant number of free electrons and radius, $r \propto t^{m}$, EM would be $\propto t^{-5 m}$. We determined the expansion rate of SN 1986J from the sequence of VLBI images and found that $m=0.69 \pm 0.03$ [5]. The time-dependence of the EM implies a slightly more decelerated expansion with $r \propto t^{-0.54 \pm 0.05}$, suggesting perhaps some on-going fragmentation in the ejecta, which would cause the opacity to not scale simply with radius.

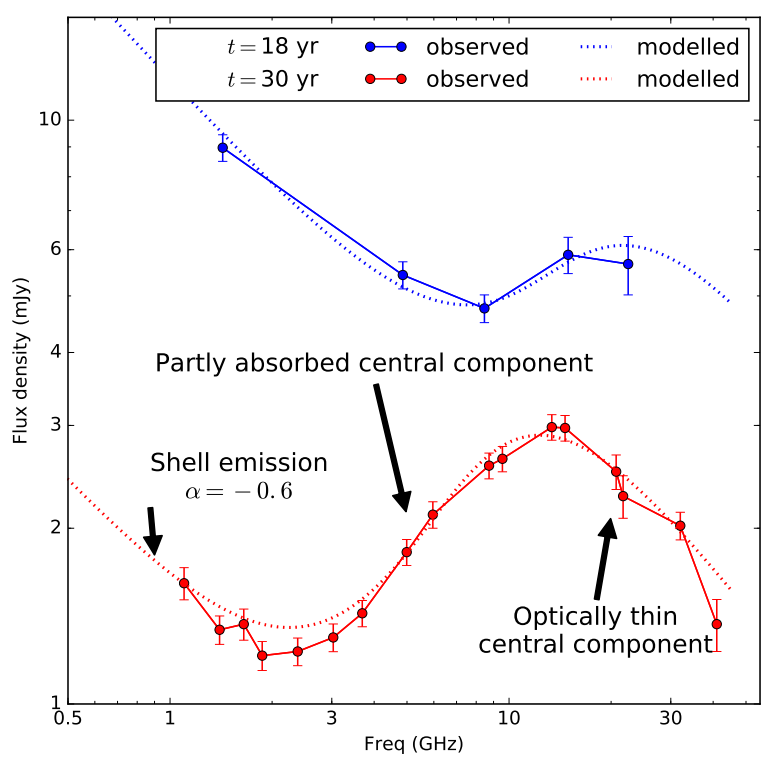

Figure 2: The evolving SED of SN 1986J. We show only two example SEDs, one from $t=18 \mathrm{yr}$ and the latest one from $t=30 \mathrm{yr}$. For SEDs at intermediate times showing the evolution in more detail, see [7]. We indicate the parts of the SED due to the central component and to the optically-thin shell on the lower curve. The uncertainties are estimated standard errors, with statistical and systematic contributions added in quadrature. The dotted lines show our Bayesian fit of a simple two-component model (one for the shell, one for the central component) to the evolving SED. See text and [7] for details. 
Extrapolating our fitted time-dependence of EM, we can calculate the age at which the 1$\mathrm{GHz}$ optical depth would be unity, $t_{\tau=1}=200 \mathrm{yr}$. Given that our model of the SED was only approximate, the extrapolation from the observations at $t=30 \mathrm{yr}$ to $200 \mathrm{yr}$ is rather uncertain. However, given the present high optical depth at $1 \mathrm{GHz}$, it is clear that the ejecta would not become transparent at $1 \mathrm{GHz}$ for at least several decades from now, so we can probably set a lower bound of $t_{\tau=1}>60 \mathrm{yr}$.

At $t=20 \mathrm{yr}$, the fitted EM is $1.64 \times 10^{9} \mathrm{~cm}^{-6} \mathrm{pc}$. At that time the radius of SN 1986J, $r_{\mathrm{SN}}=6 \times 10^{17} \mathrm{~cm}$, and the radius of the reverse shock was $\sim 4.3 \times 10^{17} \mathrm{~cm}$ [5]. If we take the free electrons to be uniformly distributed, we can calculate a total ionized mass, $M_{\mathrm{ion}} \gtrsim 40 \mathrm{M}_{\odot}$. Such a high value of $M_{\text {ion }}$ is not reasonable. We can conclude therefore that the ionized absorbing material must be non-uniformly distributed.

A non-uniform distribution of $M_{\text {ion }}$ is not unexpected: in a $\mathrm{SN}$, a dual shock structure will form with a forward shock being driven into the CSM and a reverse shock being driven back into the ejecta, with the thickness of the region between the shocks being $\sim 20 \%$ of the forward-shock radius (e.g. [13]). The material between the two shocks is at sufficiently low density and high temperatures $\left(T_{e}>10^{6} \mathrm{~K} ;[13,14]\right)$ that it does not contribute significantly to the radio absorption, or to the EM or DM [12].

The unshocked ejecta interior to the reverse shock are expected to be ionized by the SN shock breakout, but may recombine fairly rapidly thereafter. The radiation from the two shocks is likely to keep some region towards the outer radius ionized. In the specific case of SN 1986J, radiation from the central component, whatever its physical nature, may also keep some region near the centre ionized. With reasonable values of $M_{\mathrm{ion}} \leq 5 \mathrm{M}_{\odot}, \mathrm{EM}=1.64 \times 10^{9} \mathrm{~cm}^{-6} \mathrm{pc}$ can be achieved either with a region near the centre with a radius $<0.4 r_{\mathrm{SN}}$, or with a thin shell of ionized material near the reverse shock, with thickness $0.002 r_{\mathrm{SN}}$. We consider the concentration of $M_{\text {ion }}$ in such a thin shell unlikely, so it is more likely that most of the absorption comes from dense ionized ejecta near the centre of the SN.

One of the distinguishing features of FRBs are their high DMs. Can we say anything about the DM produced as signals propagate through $\mathrm{SN}$ ejecta? At our extrapolated time of transparency at $1 \mathrm{GHz}, t_{\tau=1} \simeq 200 \mathrm{yr}$, the EM would be $3 \times 10^{6} \mathrm{~cm}^{-6} \mathrm{pc}$, and the extrapolated $r_{\mathrm{SN}}$ would be $\sim 0.9 \mathrm{pc}$ [5]. For a shell of material ionized by the shocks, the shell must be very thin to be of reasonable mass $\left(<5 \mathrm{M}_{\odot}\right)$, and the DM remains $<50 \mathrm{~cm}^{-3} \mathrm{pc}$. Larger DMs would occur if the ionized material is near the centre of the $\mathrm{SN}$, ionized by some central source. In this case, an ionized region of $M_{\text {ion }}=5 \mathrm{M}_{\odot}$ of radius $\sim 0.2 r_{\mathrm{SN}}$ or $\sim 0.2 \mathrm{pc}$, would produce a DM $\sim 800 \mathrm{~cm}^{-3} \mathrm{pc}$, which is in the range observed for FRBs (more details on the calculation of the DM can be found in [12].

\section{Conclusions}

On the basis of our VLBI and VLA observations of the central component of the Type II SN 1986J, we can conclude that it is still opaque to $1 \mathrm{GHz}$ radiation $30 \mathrm{yr}$ after the explosion, and will likely remain so for at least another $30 \mathrm{yr}$. Therefore, If FRBs do originate in young neutron stars or magnetars from SNe like SN 1986J, we would not see them until at least several decades after the SN explosion. By the time the ejecta have become transparent at $1 \mathrm{GHz}$, the contribution 
of the ejecta to the DM is likely only a fraction of the values typically seen for FRBs, unless the ejecta are ionized from the centre.

Looking ahead, the Square Kilometre Array will massively boost the sensitivity of VLBI observations. It will therefore allow VLBI observations of SNe to be extended much longer than is possible at present, and will likely make it possible to resolve $\mathrm{SNe}$ at ages of several decades when they have become transparent to FRBs. The possibility of imaging FRB-engines is an exciting prospect for VLBI with the SKA.

\section{Acknowledgements}

The author acknowledges financial support from the "VLBI with the SKA" work package is part of the Jumping JIVE project, that has received funding from the European Union's Horizon 2020 research and innovation programme under grant agreement No 730884.

\section{References}

[1] J. I. Katz, Fast radio bursts - A brief review: Some questions, fewer answers, Modern Physics Letters A 31 (2016) $1630013[1604.01799]$.

[2] E. Petroff, E. D. Barr, A. Jameson, E. F. Keane, M. Bailes, M. Kramer et al., FRBCAT: The Fast Radio Burst Catalogue, PASA 33 (2016) e045 [1601.03547].

[3] S. Chatterjee, C. J. Law, R. S. Wharton, S. Burke-Spolaor, J. W. T. Hessels, G. C. Bower et al., A direct localization of a fast radio burst and its host, Nat 541 (2017) 58 [1701.01098].

[4] M. F. Bietenholz, N. Bartel and M. P. Rupen, SN 1986J VLBI: The Evolution and Deceleration of the Complex Source and a Search for a Pulsar Nebula, ApJ 581 (2002) 1132.

[5] M. F. Bietenholz, N. Bartel and M. P. Rupen, Supernova 1986J Very Long Baseline Interferometry. II. The Evolution of the Shell and the Central Source, ApJ 712 (2010) 1057.

[6] M. F. Bietenholz and N. Bartel, SN 1986J VLBI. III. The Central Component Becomes Dominant, ApJ 839 (2017) $10[1701.08447]$.

[7] M. F. Bietenholz and N. Bartel, SN 1986J VLBI. IV. The Nature of the Central Component, ApJ 851 (2017) 7 [1707.06596].

[8] N. Bartel, I. I. Shapiro and M. R. Rupen, VLBI observations of SN 1986 - Deviations from spherical symmetry in its radio brightness distribution, ApJL 337 (1989) L85.

[9] M. F. Bietenholz, N. Bartel and M. P. Rupen, Discovery of a Compact Radio Component in the Center of Supernova 1986J, Science 304 (2004) 1947.

[10] M. P. Rupen, J. H. van Gorkom, G. R. Knapp, J. E. Gunn and D. P. Schneider, Observations of SN 1986J in NGC 891, AJ 94 (1987) 61.

[11] K. W. Weiler, N. Panagia and R. A. Sramek, Radio emission from supernovae. II. SN 1986J: A different kind of type II, ApJ 364 (1990) 611.

[12] M. F. Bietenholz and N. Bartel, On the Possibility of Fast Radio Bursts from Inside Supernovae: The Case of SN 1986J, ApJ 851 (2017) 124 [1707.07746].

[13] R. A. Chevalier, The radio and X-ray emission from type II supernovae, ApJ 259 (1982) 302.

[14] P. Lundqvist and C. Fransson, Circumstellar absorption of UV and radio emission from supernovae, Astron. Astrophys. 192 (1988) 221. 